PROCEEDINGS OF THE

AMERICAN MATHEMATICAL SOCIETY

Volume 127, Number 2, February 1999, Pages 577-581

S 0002-9939(99)04513-X

\title{
OPEN COVERS AND PARTITION RELATIONS
}

\author{
MARION SCHEEPERS
}

(Communicated by Andreas R. Blass)

\begin{abstract}
An open cover of a topological space is said to be an $\omega$-cover if there is for each finite subset of the space a member of the cover which contains the finite set, but the space itself is not a member of the cover. We prove theorems which imply that a set $X$ of real numbers has Rothberger's property $\mathrm{C}^{\prime \prime}$ if, and only if, for each positive integer $k$, for each $\omega$-cover $\mathcal{U}$ of $X$, and for each function $f:[\mathcal{U}]^{2} \rightarrow\{1, \ldots, k\}$ from the two-element subsets of $\mathcal{U}$, there is a subset $\mathcal{V}$ of $\mathcal{U}$ such that $f$ is constant on $[\mathcal{V}]^{2}$, and each element of $X$ belongs to infinitely many elements of $\mathcal{V}$ (Theorem 1 ).

A similar characterization is given of Menger's property for sets of real numbers (Theorem 6).
\end{abstract}

Gerlits and Nagy [3] introduced the notion of an $\omega$-cover as defined above in the abstract. Let $\Omega$ be the set of all $\omega$-covers of $X$. These authors call $X$ an $\epsilon$-space if each $\omega$-cover has a countable subset which is an $\omega$-cover. All separable metric spaces are $\epsilon$-spaces. An open cover $\mathcal{U}$ of $X$ is said to be a large cover if for each $x \in X$ the set $\{U \in \mathcal{U}: x \in U\}$ is infinite. Let $\Lambda$ be the set of all large covers and $\mathcal{O}$ the set of all open covers of $X$.

For collections $\mathcal{A}$ and $\mathcal{B}$ of subsets of the set $S$ the symbol $\mathrm{S}_{1}(\mathcal{A}, \mathcal{B})$ denotes the property that there is for each sequence $\left(O_{n}: n=1,2,3, \ldots\right)$ of elements of $\mathcal{A}$ a sequence $\left(T_{n}: n=1,2,3, \ldots\right)$ such that for each $n T_{n} \in O_{n}$, and $\left\{T_{n}\right.$ : $n=1,2,3, \ldots\} \in \mathcal{B}$. Associated with this property we have the game $\mathrm{G}_{1}(\mathcal{A}, \mathcal{B})$ : Two players, ONE and TWO, play an inning per positive integer. In the $n$-th inning ONE chooses a set $O_{n} \in \mathcal{A}$, and TWO responds with $T_{n} \in O_{n}$. A play $\left(O_{1}, T_{1}, O_{2}, T_{2}, \ldots\right)$ is won by TWO if $\left\{T_{n}: n=1,2,3, \ldots\right\}$ is in $\mathcal{B}$; otherwise, ONE wins.

In the paper [9] where he introduced it, Rothberger used the symbol $C^{\prime \prime}$ to denote the property $S_{1}(\mathcal{O}, \mathcal{O})$. Galvin introduced the game $\mathrm{G}_{1}(\mathcal{O}, \mathcal{O})$ in [2] and Pawlikowski proved in [7]:

Theorem 1 (Pawlikowski). $X$ has property $S_{1}(\mathcal{O}, \mathcal{O})$ if, and only if, ONE does not have a winning strategy in the game $G_{1}(\mathcal{O}, \mathcal{O})$.

For $\mathcal{A}$ and $\mathcal{B}$ as above the symbol $\mathrm{S}_{\text {fin }}(\mathcal{A}, \mathcal{B})$ denotes the property that there is for each sequence $\left(O_{n}: n=1,2,3, \ldots\right)$ of elements of $\mathcal{A}$ a sequence $\left(T_{n}: n=\right.$ $1,2,3, \ldots)$ such that for each $n T_{n}$ is a finite subset of $O_{n}$, and $\bigcup_{n=1}^{\infty} T_{n} \in \mathcal{B}$.

Received by the editors April 15, 1996 and, in revised form, May 16, 1997.

1991 Mathematics Subject Classification. Primary 03E05, 05D10.

Key words and phrases. Ramsey's theorem, Rothberger's property, Menger's property, infinite game, partition relation.

The author's research was funded in part by NSF grant DMS 95-05375.

(C)1999 American Mathematical Society 
The game $\mathrm{G}_{f i n}(\mathcal{A}, \mathcal{B})$ associated with this property is as follows: Two players, ONE and TWO, play an inning per positive integer. In the $n$-th inning ONE chooses a set $O_{n} \in \mathcal{A}$, and TWO responds with a finite subset $T_{n}$ of $O_{n}$. A play $\left(O_{1}, T_{1}, O_{2}, T_{2}, \ldots\right)$ is won by TWO if $\bigcup_{n=1}^{\infty} T_{n}$ is in $\mathcal{B}$; otherwise, ONE wins.

In [4] Hurewicz showed that $S_{\text {fin }}(\mathcal{O}, \mathcal{O})$ is equivalent to a property which was introduced in [6] by Menger. The property $\mathrm{S}_{f i n}(\mathcal{O}, \mathcal{O})$ is called Menger's property to distinguish it from another property which was also introduced by Hurewicz, and called Hurewicz's property. In that same paper Hurewicz implicitly studied the game $\mathrm{G}_{f i n}(\mathcal{O}, \mathcal{O})$. Telgársky later made this game explicit in [13]. Hurewicz proved in Theorem 10 of [4]:

Theorem 2 (Hurewicz). The space $X$ has property $S_{\text {fin }}(\mathcal{O}, \mathcal{O})$ if, and only if, ONE does not have a winning strategy in $G_{\text {fin }}(\mathcal{O}, \mathcal{O})$.

It was shown in [5] and [10] through [12] that Rothberger's property, Menger's property and some other covering properties of topological spaces are intimately connected with Ramsey theory. The following two Ramseyan partition relations are relevant for our paper. Let collections $\mathcal{A}, \mathcal{B}_{1}, \ldots, \mathcal{B}_{k}$ of subsets of $S$ and positive integer $n$ be given. The first of the two partition relations we are interested in is denoted by the symbol

$$
\mathcal{A} \rightarrow\left(\mathcal{B}_{1}, \ldots, \mathcal{B}_{k}\right)^{n}
$$

This symbol means that for each $A \in \mathcal{A}$ and for each $f:[A]^{n} \rightarrow\{1, \ldots, k\}$ there are an $i \in\{1, \ldots, k\}$ and a subset $B$ of $A$ such that $B \in \mathcal{B}_{i}$ and $f(T)=i$ whenever $T \in[B]^{n}$. The subset $B$ of $A$ is said to be homogeneous for $f$. If each $\mathcal{B}_{i}$ is equal to the class $\mathcal{B}$, then we abbreviate the symbol as $\mathcal{A} \rightarrow(\mathcal{B})_{k}^{n}$. Ramsey's famous theorem in [8] essentially states for $\mathcal{A}$ the collection of infinite subsets of the positive integers that for all $n$ and $k, \mathcal{A} \rightarrow(\mathcal{A})_{k}^{n}$. The partition relation $\Omega \rightarrow(\Lambda)_{k}^{2}$ means that for each $\omega$-cover $\mathcal{U}$ of $X$ and for each function $f:[\mathcal{U}]^{2} \rightarrow\{1,2, \ldots, k\}$ there are a large cover $\mathcal{V} \subset \mathcal{U}$ of $X$ and a $j \in\{1,2, \ldots, k\}$ such that for each $\{A, B\} \in[\mathcal{V}]^{2}$, $f(\{A, B\})=j$.

The second partition relation is denoted by the symbol

$$
\mathcal{A} \rightarrow((\mathcal{B}))_{k}^{2} .
$$

This symbol means that for each $A \in \mathcal{A}$ and for each $f:[A]^{2} \rightarrow\{1, \ldots, k\}$, there are an element $B \in \mathcal{B}$, an $i \in\{1, \ldots, k\}$ and a finite-to-one function $g$ with domain $B$ such that $f(\{b, c\})=i$ whenever $g(b) \neq g(c)$. We say that $B$ is eventually homogeneous for $f$. This partition relation (but not the notation) was introduced in [1], where the authors showed that an ultrafilter $\mathcal{U}$ on the set of positive integers is a $\mathrm{P}$-point ultrafilter if, and only if, for all $k$ it satisfies the partition relation $\mathcal{U} \rightarrow((\mathcal{U}))_{k}^{2}$.

The Ramseyan partition relations given in [10] through [12] for Rothberger's property and for Menger's property all stopped short of finding a big homogeneous set for the partitions, but gave big end-homogeneous (eventually end-homogeneous in case of Menger's property) sets. We now cure this shortcoming.

\section{ROTHBERGER'S PROPERTY}

By the proof of Theorem 17 of [10] $X$ has property $S_{1}(\Lambda, \Lambda)$ if, and only if, it has property $\mathrm{S}_{1}(\mathcal{O}, \mathcal{O})$. Right after Theorem 14 of [10] I stated the following analogue of Pawlikowski's theorem without proof: 
Theorem 3. $X$ has property $S_{1}(\Lambda, \Lambda)$ if, and only if, ONE does not have a winning strategy in the game $\mathrm{G}_{1}(\Lambda, \Lambda)$.

Proof. It is easy to see that if ONE has no winning strategy in $\mathrm{G}_{1}(\Lambda, \Lambda)$, then $X$ has property $\mathrm{S}_{1}(\Lambda, \Lambda)$.

Suppose that $X$ has property $\mathrm{S}_{1}(\Lambda, \Lambda)$. We must show that ONE does not have a winning strategy in $\mathrm{G}_{1}(\Lambda, \Lambda)$. Let $F$ be a strategy for ONE. Since $X$ has property $\mathrm{S}_{1}(\Lambda, \Lambda)$, we may assume that $F$ calls on ONE to choose countable large open covers. For each countable large open cover $\mathcal{U}$ fix, in advance, a bijective enumeration $\left(\phi_{n}(\mathcal{U}): n \in \mathbb{N}\right)$.

For each $n \in \mathbb{N}$ define: $X_{n}:=X \times\{n\}$. Then the $X_{n}$ 's are disjoint. With $\tau$ denoting the topology of $X$, each $\tau_{n}:=\{U \times\{n\}: U \in \tau\}$ is a topology for $X_{n}$. The set $\bigcup_{n=1}^{\infty} \tau_{n}$ generates a topology on $\bigcup_{n=1}^{\infty} X_{n}$. Let $\sum_{n=1}^{\infty} X_{n}$ denote this space. Every open cover of $\sum_{n=1}^{\infty} X_{n}$ has a refinement which consists of elements of $\bigcup_{n=1}^{\infty} \tau_{n}$ only. If $X$ has property $\mathrm{S}_{1}(\mathcal{O}, \mathcal{O})$, so does each $\left(X_{n}, \tau_{n}\right)$. $X$ has property $\mathrm{S}_{1}(\mathcal{O}, \mathcal{O})$ if, and only if, $\sum_{n=1}^{\infty} X_{n}$ does.

Define a strategy $G$ for ONE of $\mathrm{G}_{1}(\mathcal{O}, \mathcal{O})$ on $\sum_{n=1}^{\infty} X_{n}$ as follows: ONE's first move is $G\left(\sum_{n=1}^{\infty} X_{n}\right):=\left\{\phi_{m}(F(X)) \times\{n\}: m \geq n \geq 1\right\}$. For $\underline{T}_{1}$ in $G\left(\sum_{n=1}^{\infty} X_{n}\right)$ fix $m_{1}$ and $n_{1}$ such that $\underline{T}_{1}=\phi_{m_{1}}(F(X)) \times\left\{n_{1}\right\}$, and put $T_{1}=\phi_{m_{1}}(F(X))$. Define $G\left(\underline{T}_{1}\right)$ to be $\left\{\phi_{m}\left(F\left(T_{1}\right)\right) \times\{n\}: m \geq n \geq 1\right.$ and $\left.\phi_{m}\left(F\left(T_{1}\right)\right) \neq T_{1}\right\}$. Let $\underline{T}_{2}$ be an element of $G\left(\underline{T}_{1}\right)$ and choose $m_{2}$ and $n_{2}$ such that $\underline{T}_{2}=\phi_{m_{2}}\left(F\left(T_{1}\right)\right) \times\left\{n_{2}\right\}$. Put $T_{2}=\phi_{m_{2}}\left(F\left(T_{1}\right)\right)$ and define $G\left(\underline{T}_{1}, \underline{T}_{2}\right)$ to be

$$
\left\{\phi_{m}\left(F\left(T_{1}, T_{2}\right)\right) \times\{n\}: m \geq n \geq 1 \text { and } \phi_{m}\left(F\left(T_{1}, T_{2}\right)\right) \notin\left\{T_{1}, T_{2}\right\}\right\},
$$

and so on.

By Pawlikowski's theorem $G$ is not a winning strategy. Choose a play

$$
G\left(\sum_{n=1}^{\infty} X_{n}\right), \underline{T}_{1}, \ldots, G\left(\underline{T}_{1}, \ldots, \underline{T}_{n}\right), \underline{T}_{n+1}, \ldots
$$

which defeats $G$. Then $\left\{\underline{T}_{k}: k \in \mathbb{N}\right\}$ is a cover of $\sum_{n=1}^{\infty} X_{n}$. By unravelling the definition of $G$ we find sequences $T_{1}, T_{2}, \ldots, T_{k}, \ldots, m_{1}, m_{2}, \ldots, m_{k}, \ldots$ and $n_{1}, n_{2}, \ldots, n_{k}, \ldots$ such that $T_{1}=\phi_{m_{1}}(F(X))$ and for each $k: m_{k} \geq n_{k}, T_{k+1}=$ $\phi_{m_{k+1}}\left(F\left(T_{1}, \ldots, T_{k}\right)\right), \underline{T}_{k}=T_{k} \times\left\{n_{k}\right\}$, and $T_{k+1} \notin\left\{T_{1}, \ldots, T_{k}\right\}$. Then $\left\{T_{k}: k \in \mathbb{N}\right\}$ is a large cover of $X$ and $F$ is defeated.

Following [3] we call a space an $\epsilon$-space if each open $\omega$-cover has a countable subset which is an $\omega$-cover. A space is metacompact if each open cover has an open point-finite refinement. In the proof of the following theorem we use the fact that, if an $\omega$-cover is partitioned into finitely many subfamilies, then at least one of these pieces is also an $\omega$-cover.

Theorem 4. For a metacompact $\epsilon$-space $X$ with no isolated points, the following are equivalent:

1. $X$ has property $S_{1}(\mathcal{O}, \mathcal{O})$.

2. For each positive integer $k, \Omega \rightarrow(\Lambda)_{k}^{2}$.

3. $\Omega \rightarrow(\Lambda, \text { not point-finite })^{2}$.

Proof. Towards proving $1 \Rightarrow 2$, let $\mathcal{U}$ be an $\omega$-cover of $X$, let $k$ be a positive integer and let $f:[\mathcal{U}]^{2} \rightarrow\{1,2, \ldots, k\}$ be a coloring. We may assume that $\mathcal{U}$ is countable. Enumerate it bijectively as $\left(U_{n}: n<\omega\right)$, and recursively choose an $\omega$-sequence $\left(\mathcal{U}_{0}, i_{0}\right),\left(\mathcal{U}_{1}, i_{1}\right), \ldots$ such that $\mathcal{U}_{0}=\left\{U_{n} \in \mathcal{U}: n>0\right.$ and $\left.f\left(\left\{U_{0}, U_{n}\right\}\right)=i_{0}\right\}$ is an 
$\omega$-cover of $X$, and for each $m \mathcal{U}_{m+1}=\left\{U_{n} \in \mathcal{U}_{m}: n>m+1\right.$ and $f\left(\left\{U_{m+1}, U_{n}\right\}\right)=$ $\left.i_{m+1}\right\}$ is an $\omega$-cover.

For each $j \in\{1, \ldots, k\}$ define $\mathcal{W}_{j}=\left\{U_{n} \in \mathcal{U}: i_{n}=j\right\}$. Then for each $n$ $\mathcal{U}_{n} \cap \mathcal{W}_{1}, \ldots, \mathcal{U}_{n} \cap \mathcal{W}_{k}$ is a partition of the $\omega$-cover $\mathcal{U}_{n}$ into $k$ disjoint parts; choose $j_{n}$ such that $\mathcal{U}_{n} \cap \mathcal{W}_{j_{n}}$ is an $\omega$-cover. Then there is a fixed $j$ such that for infinitely many $n$ we have $j_{n}=j$. Since the sequence of $\mathcal{U}_{n}$ 's is decreasing, this means that for each $n \mathcal{U}_{n} \cap \mathcal{W}_{j}$ is an $\omega$-cover of $X$.

Now define a strategy $\sigma$ for ONE in the game $G_{1}(\Lambda, \Lambda)$ as follows: Let $n_{0}$ be minimal such that $U_{n_{0}} \in \mathcal{U}_{0} \cap \mathcal{W}_{j}$, and let ONE's first move be $\sigma(X)=\mathcal{U}_{n_{0}} \cap \mathcal{W}_{j}$. If TWO responds to this with $U_{n_{1}} \in \sigma(X)$, then ONE plays $\sigma\left(U_{n_{1}}\right)=\mathcal{U}_{n_{1}} \cap \mathcal{W}_{j}$. If TWO now chooses $U_{n_{2}} \in \sigma\left(U_{n_{1}}\right)$, then ONE plays $\sigma\left(U_{n_{1}}, U_{n_{2}}\right)=\mathcal{U}_{n_{2}} \cap \mathcal{W}_{j}$, and so on.

Since $\sigma$ is not a winning strategy for ONE, let $\sigma(X), U_{n_{1}}, \sigma\left(U_{n_{1}}\right), U_{n_{2}}, \sigma\left(U_{n_{1}}, U_{n_{2}}\right)$, $\ldots$ be a play lost by ONE. Then $\left\{U_{n_{1}}, U_{n_{2}}, \ldots\right\}$ is a large cover for $X$ such that for each $t n_{t}<n_{t+1}$ and $U_{n_{t+1}} \in \mathcal{U}_{n_{t}} \cap \mathcal{W}_{j}$. Then $\left\{U_{n_{1}}, U_{n_{2}}, \ldots\right\}$ is homogeneous of color $j$ for $f$.

$2 \Rightarrow 3$ : No large cover is point-finite. Thus, 3 follows from 2 .

$3 \Rightarrow 1$ : In Theorem 17 of [10] it was proved that $S_{1}(\mathcal{O}, \mathcal{O})$ is equivalent to $\mathrm{S}_{1}(\Omega, \mathcal{O})$. We show that the partition relation of 3 implies $\mathrm{S}_{1}(\Omega, \mathcal{O})$.

Let $\left(\mathcal{U}_{n}: n<\omega\right)$ be a sequence of $\omega$-covers of $X$. By Lemma 3 of [10] choose for each $n$ a point-finite open refinement $\mathcal{W}_{n}$ of $\mathcal{U}_{n}$ such that $\mathcal{W}_{m} \cap \mathcal{W}_{n}=\emptyset$ whenever $m \neq n$ (here we use " $X$ is metacompact with no isolated points"). Also choose a partition $\left(Y_{n}: n \in \mathbb{N}\right)$ of $\omega$ into disjoint sets such that $Y_{n}$ has cardinality $n$.

For each $n$ put $\mathcal{R}_{n}:=\left\{S_{j_{1}} \cup \cdots \cup S_{j_{n}}: S_{j_{i}} \in \mathcal{W}_{j_{i}}\right.$ and $\left.j_{i} \in Y_{n}\right\}$ (the $j_{i}$ 's are taken to be distinct). Each $\mathcal{R}_{n}$ is a point-finite cover of $X$. We may assume that $X$ is not in any of the $\mathcal{R}_{n}$ 's. Let $\mathcal{U}$ be the $\omega$-cover $\bigcup_{n=1}^{\infty} \mathcal{R}_{n}$ of $X$. For each $U \in \mathcal{U}$ fix an $f(U)$ such that $U \in \mathcal{R}_{f(U)}$. Define a partition $\phi:[\mathcal{U}]^{2} \rightarrow\{0,1\}$ so that

$$
\phi(\{U, V\})= \begin{cases}0 & \text { if } f(U) \neq f(V), \\ 1 & \text { otherwise. }\end{cases}
$$

Since homogeneous sets of color 1 for $\phi$ are point-finite, the partition hypothesis gives a subset $\mathcal{W}$ of $\mathcal{R}$ which is homogeneous of color 0 and is a large cover of $X$. By dismantling the elements of $\mathcal{W}$ we find a sequence of sets with for each $n$ the $n$-th term from $\mathcal{W}_{n}$, such that this sequence covers $X$. Then for each $n$ choose a set from $\mathcal{U}_{n}$ which contains the $n$-th term of this sequence.

\section{MENGER'S PROPERTY}

In [5] it was shown that having property $\mathrm{S}_{\text {fin }}(\mathcal{O}, \mathcal{O})$ is equivalent to having property $S_{f i n}(\Lambda, \Lambda)$. Using ideas in the proof of Theorem 3 and Hurewicz's theorem instead of Pawlikowski's one can show:

Theorem 5. The space $X$ has property $S_{\text {fin }}(\Lambda, \Lambda)$ if, and only if, ONE does not have a winning strategy in $G_{f i n}(\Lambda, \Lambda)$.

By the methods of [10] $X$ has Menger's property if, and only if, it has property $\mathrm{S}_{\text {fin }}(\Omega, \Lambda)$. Using this and Theorem 5 we obtain:

Theorem 6. For any $\epsilon$-space $X$ the following are equivalent:

1. $X$ has Menger's property.

2. For each positive integer $k, \Omega \rightarrow((\Lambda))_{k}^{2}$. 
Proof. The implication $2 \Rightarrow 1$ is proved like the implication $3 \Rightarrow 1$ of Theorem 4 . To prove $1 \Rightarrow 2$ we proceed as in the proof of $1 \Rightarrow 2$ of Theorem 4 : From the countable $\omega$-cover $\mathcal{U}$ and the coloring $f:[\mathcal{U}]^{2} \rightarrow\{1, \ldots, k\}$ we obtain a sequence of $\mathcal{U}_{n}$ 's and $i_{n}$ 's exactly as we did there. Then we find an $\omega$-cover $\mathcal{W}_{j}$ just as there. Now comes the difference. Instead of the game $G_{1}(\Lambda, \Lambda)$ played in the proof of Theorem 4 we now play $\mathrm{G}_{f i n}(\Lambda, \Lambda)$. By Theorem 5 ONE has no winning strategy in this game. Define a strategy $\sigma$ for ONE as follows. The first move is like the one in the proof of Theorem 4. If TWO chooses the finite subset $\left\{U_{n_{1}}, \ldots, U_{n_{k}}\right\}$ from ONE's most recent move, and this listing is in order of increasing subscripts, then ONE responds with the strategy $\sigma$ from the proof of Theorem 4 as if TWO played $U_{n_{k}}$.

A play with this strategy which is lost by ONE produces an eventually homogeneous large cover of color $j$ for $X$.

\section{REFERENCES}

1. J.E. Baumgartner and A.D. Taylor, Partition theorems and ultrafilters, Transactions of the American Mathematical Society 241 (1978), 283 - 309. MR 58:10458

2. F. Galvin, Indeterminacy of point-open games, Bulletin de L'Académie Polonaise des Sciences 26 (1978), $445-448$. MR 58:12881

3. J. Gerlits and Zs. Nagy, Some properties of C $(X)$, I, Topology and its Applications 14 (1982), $151-161$. MR 84f:54021

4. W. Hurewicz, Über die Verallgemeinerung des Borelschen Theorems, Mathematische Zeitschrift 24 (1925), $401-421$.

5. W. Just, A.W. Miller, M. Scheepers and P.J. Szeptycki, Combinatorics of open covers (II), Topology and its Applications 73 (1996), 241 - 266. CMP 97:04

6. K. Menger, Einige Überdeckungssätze der Punktmengenlehre, Sitzungsberichte der Wiener Akademie Abt. 2a, Mathematik, Astronomie, Physik, Meteorologie und Mechanik 133 (1924), 421 - 444.

7. J. Pawlikowski, Undetermined sets of Point-Open games Fundamenta Mathematicae 144 (1994), $279-285$. MR 95i:54043

8. F.P. Ramsey, On a problem of formal logic, Proceedings of the London Mathematical Society 30 (1930), $264-286$.

9. F. Rothberger, Eine Verschärfung der Eigenschaft C, Fundamenta Mathematicae 30 (1938), $50-55$.

10. M. Scheepers, The combinatorics of open covers (I): Ramsey theory, Topology and its Applications 69 (1996), 31 - 62. MR 97h:90123

11. M. Scheepers, Rothberger's property and partition relations, The Journal of Symbolic Logic 62 (1997), 976-980. CMP 98:01

12. M. Scheepers, Open covers and the square bracket partition relation, Proceedings of the American Mathematical Society 125 (1997), 2719-2724. MR 97j:04001

13. R. Telgársky, On games of Topsøe, Mathematica Scandinavica 54 (1984), $170-176)$. MR 88f:54061

Department of Mathematics, Boise State University, Boise, Idaho 83725

E-mail address: marion@math.idbsu.edu 